\title{
Simulation of the Columnar-to-Equiaxed Transition in Alloy Solidification -- the Effect of Nucleation Undercooling, Density of Nuclei in Bulk Liquid and Alloy Solidification Range on the Transition
}

\author{
H.J. Dai ${ }^{1, a}$, H. B. Dong ${ }^{1, b}$, H.V. Atkinson ${ }^{1, c}$, P.D. Lee ${ }^{2, d}$ \\ ${ }^{1}$ Department of Engineering, University of Leicester, University Road, Leicester LE1 7RH, UK \\ ${ }^{2}$ Department of Materials, Imperial College London, Exhibition Road, London SW7 2BP, UK \\ ahd44@le.ac.uk, ${ }^{b}$ hd38@le.ac.uk, ${ }^{c h}$ ha2@le.ac.uk, ${ }^{d}$ p.d.lee@imperial.ac.uk
}

Keywords: Columnar-to-equiaxed transition; Directional solidification; Computer simulation; Solidification structure.

\begin{abstract}
A coupled cellular automaton-finite difference (CA-FD) model is used to simulate the detailed dendritic structure evolution of the columnar-to-equiaxed transition (CET) for Al-Cu alloys during solidification. The effects of material properties (nucleation undercooling, density of nuclei in bulk liquid and alloy solidification range) on the CET are investigated. Simulated results reveal that: (1) equiaxed grains form at an earlier stage with a smaller critical nucleation undercooling; (2) CET is promoted if the density of nuclei in bulk liquid is increased; (3) extending the alloy solidification range promotes the CET. Finally, CET maps corresponding to different alloy concentrations are constructed, illustrating the relationship between processing conditions and the resulting grain structures for alloys with different solidification ranges.
\end{abstract}

\section{Introduction}

In castings, there are two distinct grain structures, columnar and equiaxed, which are mainly determined by material properties and solidification conditions. In direct-chill and die castings, columnar grains form near the mould surface and grow perpendicular to the isotherms, and then transform to equiaxed grains in the lower thermal gradient area near the centre of the casting. To obtain an equiaxed grain structure, inoculation of the melt with heterogeneous nuclei is widely used to promote the columnar-to-equiaxed transition (CET). Conversely, in directionally solidified or single crystal components, columnar grains are desirable, with equiaxed grains being treated as casting defects because high angle boundaries must be avoided. As a demand, high thermal gradients and low growth rates are imposed to inhibit the CET. To control the solidification structures, a better understanding on the CET is required.

CET has been studied for many years via experimental and theoretical methods. The first analytic CET model was developed by Hunt and Flood [1-3] using a mechanical blocking criterion. Their model was extended to rapid solidification conditions by Gaumann, Trivedi and Kurz [4-5] to predict the CET during welding. Recently, microstructural models (such as cellular automaton [6-8] and phase-field [9]) have been applied to investigate the effect of solidification conditions on the CET. The microstructural modeling allows individual grains to be visualized. Predicted CET for Al-Cu alloys from prior studies are summarized in Fig. 1. The figure illustrates the relationship between the processing conditions (thermal gradient and growth velocity) and solidification structure (columnar and equiaxed grains), and a reasonable agreement is obtained for the transition among different models. However, the effect of material properties on the CET has not been systematically studied using microstructural modeling.

In this study, a coupled cellular automaton-finite difference (CA-FD) microstructural model is used to simulate the detailed dendritic structure evolution and to investigate the effect of material properties (critical nucleation undercooling, density of nuclei in bulk liquid and alloy solidification range) on the CET. 


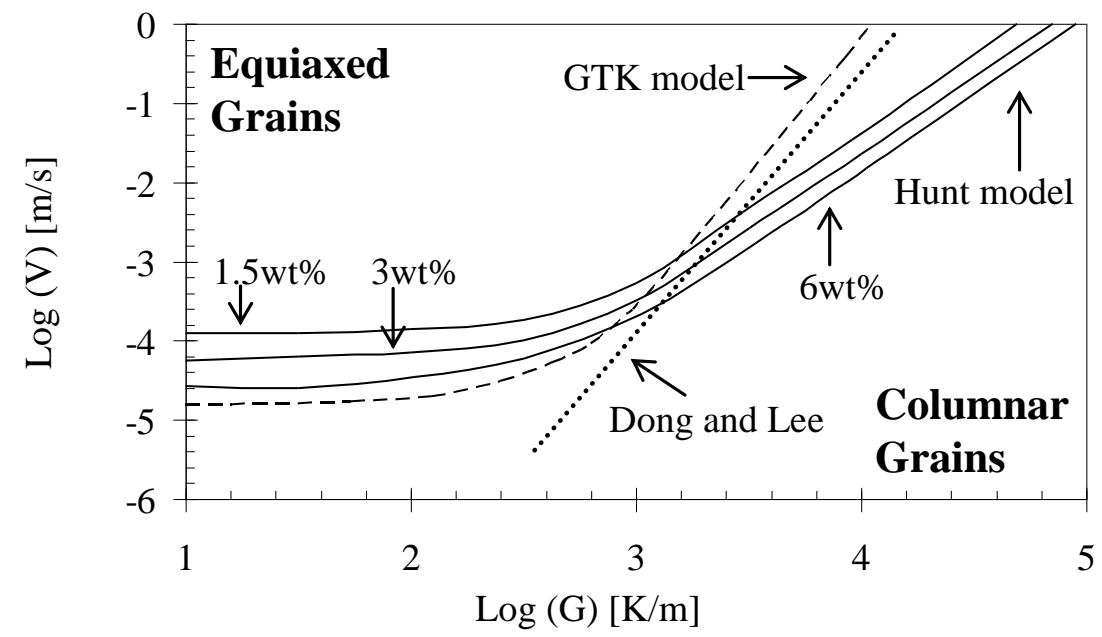

Fig. 1 Predicted columnar-to-equiaxed transition lines from prior models. The solid curves are the CET lines calculated using the Hunt model for Al-1.5wt\% Cu, Al-3wt $\% \mathrm{Cu}$ and Al-6wt $\% \mathrm{Cu}$ alloys [1]. The dashed curves are the CET lines using the GTK model [4] and Dong and Lee's predictions [8] for $\mathrm{Al}-3 \mathrm{wt} \% \mathrm{Cu}$ alloy.

\section{Model Description}

The CA-FD model is an extension of the cellular automaton technique to include solute diffusion through combination with a finite difference solver. A detailed description of the model formulation has been presented previously [10]. Therefore only the key assumptions together with the grain nucleation and growth model are summarized below.

In this model, both the CA and FD components run on the same regular spatial square grid and with the same time step. Each spatial cell represents a small amount of material, and can have three different states: liquid, solid and growing (i.e. a mixture of solid and liquid). The model begins with all cells in a liquid state, which may transform to a growing state by either nucleation or growth from its neighbouring cells. Solute is partitioned between liquid and solid phases in the interface of a growing cell. At each time step, the solute diffusion through the liquid and solid will be calculated which will affect the level of undercooling due to the change of local liquidus temperature, and then in turn affect the nucleation and growth processes.

Two types of nucleation are used in the model: pre-fixed nucleation at specific sites and stochastic nucleation in bulk liquid. Pre-fixed nucleation is used to simulate the nucleation at the bottom of the domain (mould surface). In simulation, nuclei are placed at the bottom of the domain prior to the calculation, with one of their $\langle 100\rangle$ crystallographic orientations aligned with the vertical temperature gradient. In bulk liquid, stochastic nucleation is incorporated to set up nuclei randomly with specific threshold undercooling, representing the statistical nature of the process. A continuous Gaussian nucleation distribution is used to relate the total number of nuclei in the bulk liquid with undercooling [11],

$$
n(\Delta T)=\frac{N_{M A X}}{\sqrt{2 \pi} \Delta T_{\sigma}} \int_{0}^{\Delta T} \exp \left[-\frac{\Delta T-\Delta T_{N}^{2}}{2 \Delta T_{\sigma-}^{2}}\right] d(\Delta T)
$$

Where $n(\Delta T)$ is the total number of nuclei in the bulk liquid at the undercooling $\Delta T, \Delta T_{N}$ is the mean nucleation undercooling; $\Delta T_{\sigma}$ is the standard deviation of the distribution and $N_{M A X}$ is the maximum nuclei density.

Nucleation will occur in a cell only if the cell has a randomly distributed nucleus and the undercooling in the cell is larger than the nucleation undercooling $\left(\Delta T_{N}\right)$. After nucleation, the state 
index of the cell is changed to "growing" state with a random crystallographic orientation $\theta$. Growth of the nucleated grains is controlled by solute diffusion and a modified, decentred-square growth technique [10].

Simulations presented in this paper correspond to $\mathrm{Al}-\mathrm{Cu}$ alloys, and were performed on a regular square grid with a cell edge length of $5 \mu \mathrm{m}$. Periodical boundary condition was applied to the left and right sides of the domain. The time step used was $1 \mathrm{~ms}$. Material properties and processing parameters used in the model are listed in Table 1 and the phase diagram data is generated from MTDATA [12].

Table 1 Material properties and model parameters used in the simulations

\begin{tabular}{|c|c|c|c|c|c|}
\hline \multirow[t]{2}{*}{ Parameter } & \multirow{2}{*}{$\begin{array}{l}\text { Symbol } \\
\text { [Units] }\end{array}$} & \multicolumn{4}{|c|}{ Value } \\
\hline & & $1 \mathrm{wt} \% \mathrm{Cu}$ & $3 \mathrm{wt} \% \mathrm{Cu}$ & $5 \mathrm{wt} \% \mathrm{Cu}$ & $7 \mathrm{wt} \% \mathrm{Cu}$ \\
\hline Liquidus temperature & $\mathrm{T}_{\mathrm{L}}[\mathrm{K}]$ & 930.4 & 925.4 & 920.3 & 915.3 \\
\hline Solidus temperature & $\mathrm{T}_{\mathrm{S}}[\mathrm{K}]$ & 906.5 & 863 & 829.2 & 812.2 \\
\hline Liquidus slope & $\mathrm{m}_{\mathrm{L}}[\mathrm{K} / \mathrm{wt} \%]$ & -2.653 & -2.717 & -2.729 & -2.742 \\
\hline Solidus slope & $\mathrm{m}_{\mathrm{S}}[\mathrm{K} / \mathrm{wt} \%]$ & -27.31 & -27.65 & -27.45 & -27.01 \\
\hline Partition coefficient & $\mathrm{k}$ & 0.09716 & 0.09825 & 0.09942 & 0.10105 \\
\hline Diffusion coefficient in liquid & $\mathrm{D}_{\mathrm{L}}\left[\mathrm{m}^{2} / \mathrm{s}\right]$ & \multicolumn{4}{|c|}{$3.4 \times 10^{-9}$} \\
\hline Diffusion coefficient in solid & $\mathrm{D}_{\mathrm{S}}\left[\mathrm{m}^{2} / \mathrm{s}\right]$ & \multicolumn{4}{|c|}{$D_{S}^{C u}=4.8 \times 10^{-5} \exp (-16069 / T)$} \\
\hline Gibbs Thomson Coefficient & $\Gamma[\mathrm{K} \cdot \mathrm{m}]$ & \multicolumn{4}{|c|}{$1.0 \times 10^{-7}$} \\
\hline
\end{tabular}

\section{Results and Discussion}

In this section, the influence of the nucleation criteria including critical nucleation undercooling and the density of the nuclei in bulk liquid on the CET is presented first. Then the effect of alloy solidification range on the CET is reported.

Effect of critical nucleation undercooling. The representative contour maps of simulated solute concentration with different critical nucleation undercoolings for an alloy $\mathrm{Al}-3 \mathrm{wt} \% \mathrm{Cu}$ after $18 \mathrm{~s}$ growth are shown in Fig. 2. The domain size is $3 \mathrm{~mm}$ wide by $4 \mathrm{~mm}$ high, giving a total number of $600 \times 800$ cells. Temperature gradient $(G)$ of $3.0 \mathrm{~K} / \mathrm{mm}$ was imposed to move from the bottom to the top with a pulling velocity $(V)$ using the relation: $V=100+20 \times t(\mu \mathrm{m} / \mathrm{s})$ to simulate the thermal profile of directional solidification in experiments.

To simulate the chill effect near the mould wall, more than enough seeds were prefixed at the bottom of the simulation domain before simulation. A thin layer of fine grains grew from prefixed seeds and only a few long columnar grains succeed in growing due to the competitive nature of the growth. Primary columnar dendrites grew from the bottom and secondary branches began to develop from primary arms when the space became wide in the groove. With the increase of pulling velocity, equiaxed grains formed first in the groove between the primary columnar dendrites where the undercooling is higher than the existing columnar dendrites around it and hence favours the nucleation of stray grains. The competitive growth occurred between the formed equiaxed grains and columnar dendrites. If large critical nucleation undercooling $(8 \mathrm{~K})$ is used, see Fig. 2(f), the solidification structures are constituted of columnar grains and no equiaxed grains occur; when a smaller undercooling $(7 \mathrm{~K})$ is applied, see Fig. 2 (e), the nucleated equiaxed grains can be overgrown by the columnar dendrites and act as small "islands" in the final columnar dendritic region. If the nucleation undercooling is reduced to less than $6 \mathrm{~K}$, equiaxed grains are formed in front of the columnar dendrite tips with random orientations and finally block the columnar growth, leading to the CET, see Figs. 2(a-d).

Fig. 2 illustrates the influence of critical mean nucleation undercooling $\left(\Delta T_{N}\right)$ on the CET. In Figs. 2(a) and (b), small mean undercoolings are used to simulate the inoculation effect in casting (by 
adding effective inoculant particles into melt before casting). Equiaxed grains are formed immediately from a thin layer of fine columnar grains near the surface. A reduction in grain size is also observed with the decrease of critical undercooling from Figs. 2(d) to Fig. 2(a). This agrees with prior analytical prediction [13] that inoculant particles become active at an undercooling inversely proportional to the particle diameter. In Figs. 2(c) and 2(d), the mean nucleation unsercoolings are set to $5 \mathrm{~K}$ and $6 \mathrm{~K}$. The microstructure evolution of the CET is illustrated clearly and CET occurs at a later stage with a higher growth velocity $(V=100+20 \times t(\mu \mathrm{m} / \mathrm{s}))$ when the critical nucleation undercooling increases. CET can be seen in Fig. 2(e) with a mean undercooling of $7 \mathrm{~K}$. It happens at a very late stage with a high pulling velocity until the undercooling reaches the critical limitation. In Fig. 2(f), an arbitrary high mean undercooling of $8 \mathrm{~K}$ is used to simulate solidification with difficulty in heterogeneous nucleation. In this case, no equiaxed grains can be formed within the range of velocities tested.

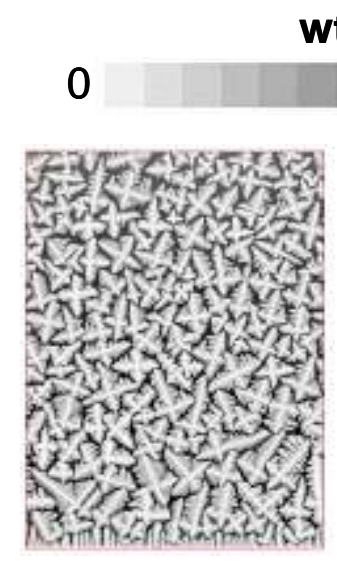

(a)

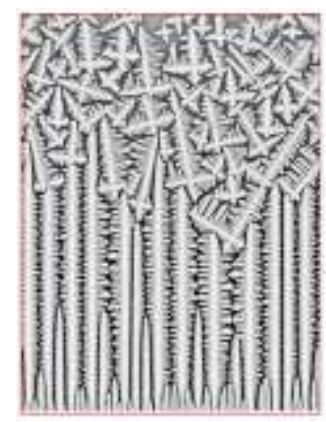

(d)

wt.\% Cu

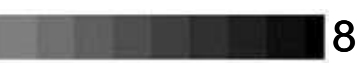

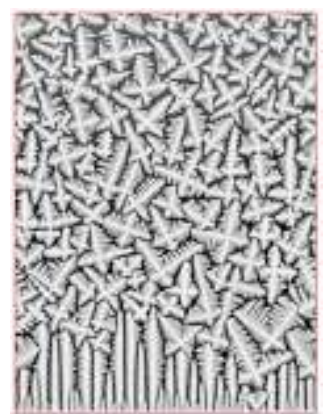

(b)

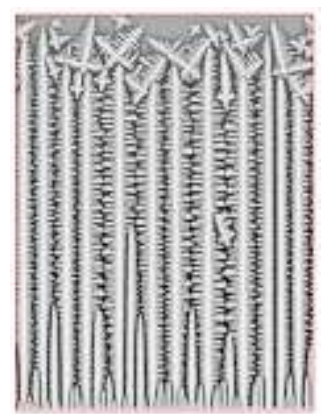

(e)

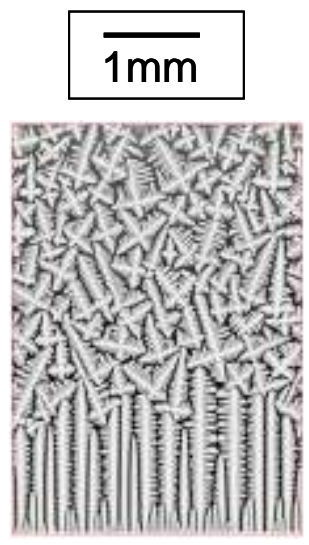

(c)

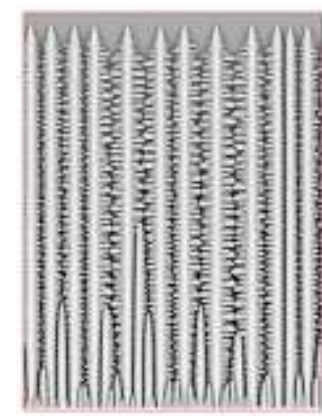

(f)

Fig. 2 Effect of critical nucleation undercooling on the CET, for a range of mean nucleation undercooling $\left(\Delta T_{N}\right)$, (a) $3 \mathrm{~K}$; (b) $4 \mathrm{~K}$; (c) $5 \mathrm{~K}$; (d) $6 \mathrm{~K}$; (e) $7 \mathrm{~K}$; (f) $8 \mathrm{~K}$

Effect of nuclei density. Different densities of nuclei in bulk liquid are used in this section to investigate the effect of nuclei density on the CET (see Fig. 3). Density of nuclei in bulk liquid changes from $6.5 \times 10^{10}$ (number of nuclei $/ \mathrm{m}^{3}$ ) (Fig. 3 (a)) to $6.5 \times 10^{14}$ (number of nuclei $/ \mathrm{m}^{3}$ ) (Fig. 3 (e)). Simulations illustrate that the CET is promoted at a lower growth velocity and higher thermal gradient for the melt with a high density of nuclei. With the increase in maximum nuclei density in bulk liquid, more equiaxed grains are nucleated when the critical undercooling is reached and in turn leads to a finer grain size in casting. This is compatible with experimental observation that inoculation of the melt with heterogeneous nuclei promotes the CET [13]. 


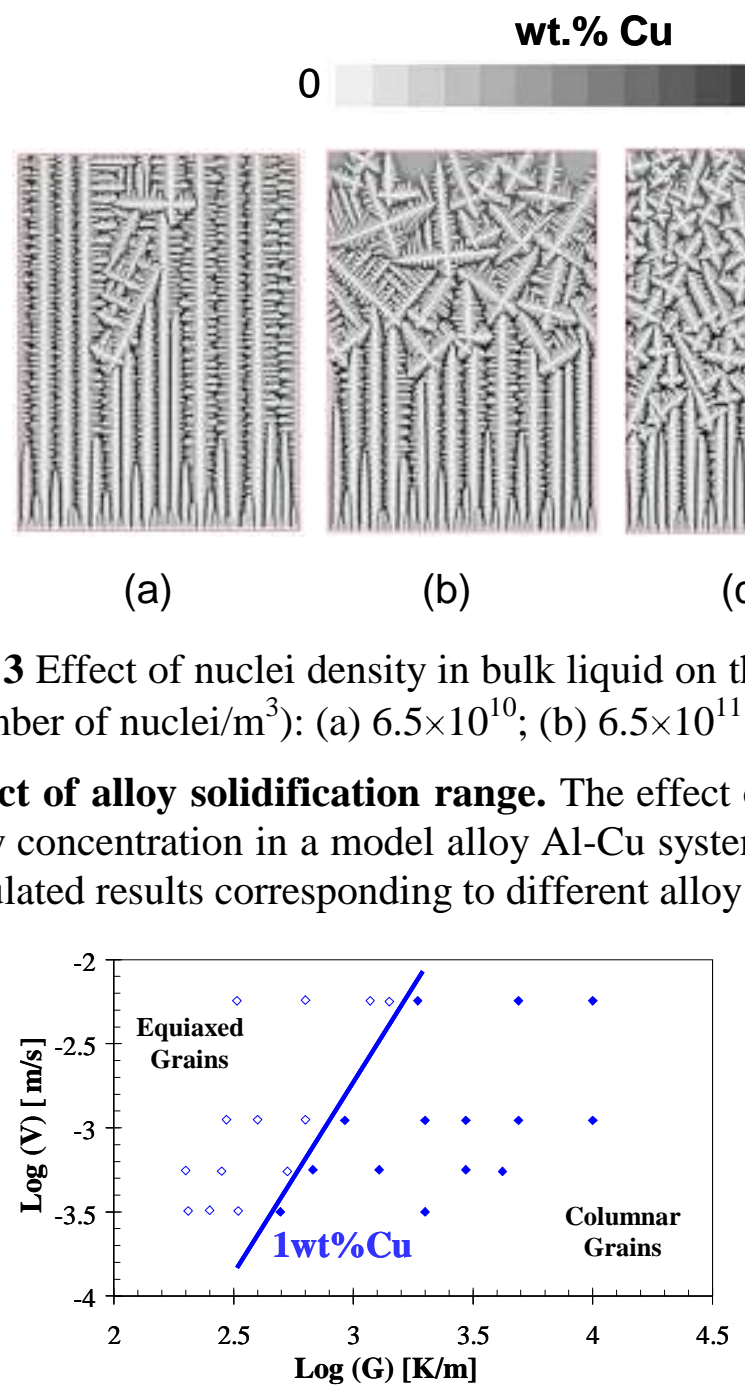

(a)

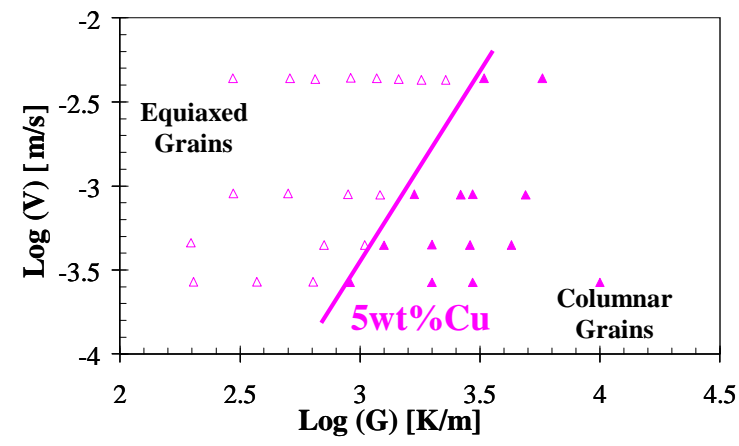

(c)

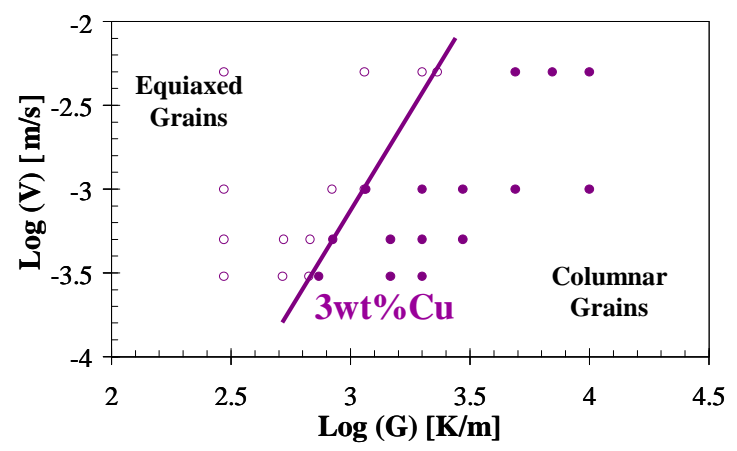

(b)

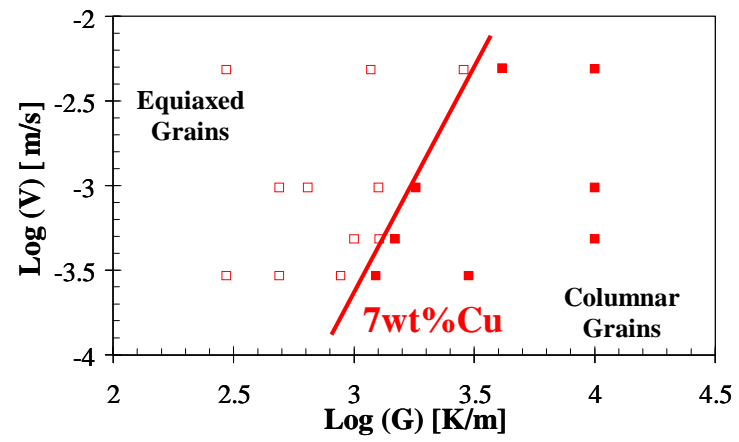

(d)

Fig. 4 Progress maps showing the effect of alloy solidification range on the CET: (a) open and filled diamonds indicate equiaxed and columnar grains for $\mathrm{Al}-1 \mathrm{wt} \% \mathrm{Cu}$; (b)open and filled circles indicate equiaxed and columnar grains for $\mathrm{Al}-3 \mathrm{wt} \% \mathrm{Cu}$; (c) open and filled triangles indicate equiaxed and columnar grains for $\mathrm{Al}-5 \mathrm{wt} \% \mathrm{Cu}$; (d) open and filled squares indicate equiaxed and columnar grains for $\mathrm{Al}-7 \mathrm{wt} \% \mathrm{Cu}$. The solid lines are approximate CET lines separating the equiaxed and columnar regions for (a) Al-1wt\%Cu;(b) $\mathrm{Al}-3 \mathrm{wt} \% \mathrm{Cu}$;(c) $\mathrm{Al}-5 \mathrm{wt} \% \mathrm{Cu}$;(d) $\mathrm{Al}-7 \mathrm{wt} \% \mathrm{Cu}$.

Simulated grains are indicated by using different symbols and the approximate CET lines separating the equiaxed and columnar regions are plotted as well. Simulated results reveal that extending the solidification range leads to an increased tendency to the CET. The reason is that for alloy with higher solute concentration, more solute will be ejected and accumulated in the solid/liquid 
interface (when $\mathrm{k}<1$ ) and in turn increase both the magnitude and the area of the constitutional undercooling ahead of the columnar front, and hence promote the CET. The simulated results match well with prior experimental observations on the CET for Al-2wt\%Cu and Al-4wt\%Cu [14].

\section{Conclusions}

Solidification structure with detailed dendritic features is simulated using the CA-FD model and the effect of material properties on the columnar-to-equiaxed transition (CET) is predicted. Simulated results reveal that the CET can be promoted by either decreasing the critical nucleation undercooling or increasing the nuclei density of the melt. Simulations also illustrate that extending the solidification range promotes the CET.

\section{Acknowledgements}

The authors would like to acknowledge funding support for computational facilities and interaction by the EPSRC (GR/T26344). H.B. Dong acknowledges the Royal Society and EPSRC for offering a Royal Society Industry Fellowship and Rolls-Royce plc for hosting him at the Precision Casting Facility (PCF), Rolls-Royce plc..

\section{References}

[1] J. D. Hunt. Mater Sci Eng 1984; 65:75.

[2] J. D. Hunt and S. C. Flood, Journal of Crystal Growth, 1987, 82, 552.

[3] J. D. Hunt and S. C. Flood, Journal of Crystal Growth, 1987, 82, 543.

[4] Gaumann M, Trivedi R, Kurz W. Mater Sci Eng A 1997;226:763.

[5] M. Gaumann, R. Trivedi and W. Kurz, Mater. Sci. Eng. A-Struct. Mater. Prop. Microstruct. Process, 1997, 226, 763.

[6] C. A. Gandin and M. Rappaz. Acta Mater 1994;42:2233.

[7] M. Vandyoussefi and A. L. Greer. Acta Mater 2002;50:1693.

[8] H.B. Dong and P.D. Lee. Acta Mater 2005;53:659

[9] A. Badillo and C. Beckermann. Acta Mater 2006;54:2015

[10] W. Wang, P. D. Lee, M. McLean. Acta Mater 2003 51:2971.

[11]C. A. Gandin, M. Rappaz and R. Tintillier, Metall. Mater. Trans. A-Phys. Metall. Mater. Sci., $1994,25,629$.

[12] R. H. Davies, A. T. Dinsdale, T. G. Chart, T. I. Barry and M. H. Rand, High Temp. Sci., 1990, 26, 251.

[13] A. L. Greer, A. M. Bunn, A. Tronche, P. V. Evans and D. J. Bristow. Acta Mater 2000, 48, 2823.

[14] C. A. Siqueira, N. Cheung, A. Garcia. Metall Mater Trans A 2002;33:2107. 